\title{
Case Report: Destructive Neuroendocrine Cranial Tumour and The Role Of Pre-Fashioned Polyetheretherketone (PEEK) Cranioplasty
}

\author{
Joseph Merola*, Thirayan Muthu, Zakier Hussain and Duncan Lamont
}

Neurosurgery Department Waikato hospital Private bag 3200, Hamilton 3240, New Zealand

\begin{abstract}
Medical implant devices have evolved in the past decade and now many surgical specialties have turned to polyetheretherketone (PEEK) devices for its ideal properties. In Neurosurgery, PEEK has its role in many operations such as cervical cages after anterior cervical discectomy and as a prosthetic implant for cranioplasty where autologous cranioplasty is not possible.
\end{abstract}

We discuss our case of a poorly differentiated destructive tumour that was surgically removed and the skull deformity replaced with a PEEK implant, assisted by state of the art neuro-navigational technology.

Keywords: Bio-compatible implants, Cranioplasty, Neuro-endocrine tumour, PEEK.

\section{INTRODUCTION}

We present the case of a 52 year old patient with a large destructive calvarial tumour who underwent excision and polyetheretherketone (PEEK) cranioplasty. Unfortunately the tumour was difficult to diagnose histologically and thought to be of neuroendocrine origin with unknown primary. Although cancers of unknown origin are relatively common, neuroendocrine tumours account for less than five percent of these (based on American figures) [1]. In 2009 the Cancer Trial New Zealand quoted an expected incidence of 250-350 neuroendocrine tumours per year in New Zealand alone with the majority reportedly presenting as metastases [2].

Calvarial metastases are not uncommon and often present with cosmetic disapproval from the patient. Treatment is surgical resection with reconstruction of eroded skull bone and imaging plays a major role in this type of surgery. Preoperatively three dimensional CT head images are obtained and are used to construct the medical implant and its template using PEEK polymer. Imaging also gives information regarding encroachment of underlying or neighbouring structures, such as dural sinuses. This is why more and more intraoperative neuro-navigational imaging is being used and allowing for safe, data-driven decisions both pre-operatively and intra-operatively.

The case is presented to raise awareness and highlight the positive impact of neuro-navigational assisted cranial reconstruction in modern times using PEEK.

\section{CASE REPORT}

A 52 year old Maori male presented to the emergency department with increasing confusion and revealed an enlarging lump on the left frontal aspect of his head. He had

\footnotetext{
*Address correspondence to this author at the Neurosurgery Department Waikato hospital Private bag 3200 Hamilton 3240 New Zealand; Tel: 02102555064; E-mail: merolaj@doctors.org.uk
}

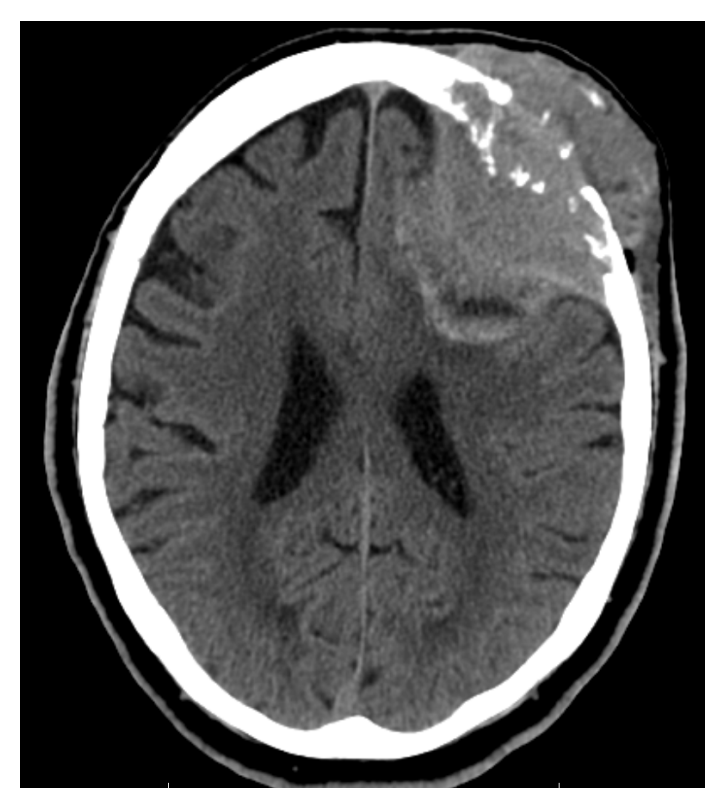

Fig. (1). CT Scan demonstrating the large frontal region, erosive mass with extension through thr tables of the skull intracranially.

noticed the lump six months prior, following a fall. He had a background of hypertension and gout with no family history of note. He was an independent, non-smoking male. There were no focal deficits elicited on examination but his wife explained that she noticed personality changes. A CT and MRI scan of the brain revealed a large mass extending intra and extra-cranially with erosion of the skull (Fig. 1, 2). A CT of the chest, abdomen and pelvis ruled out the presence of a primary lesion.

Tumour removal and cranial reconstruction was planned as a single procedure. A PEEK cranial implant was preoperatively designed based on high resolution 3D CT images. 3D CT skull images were uploaded to a medical implant manufacturing company in Switzerland. The senior 


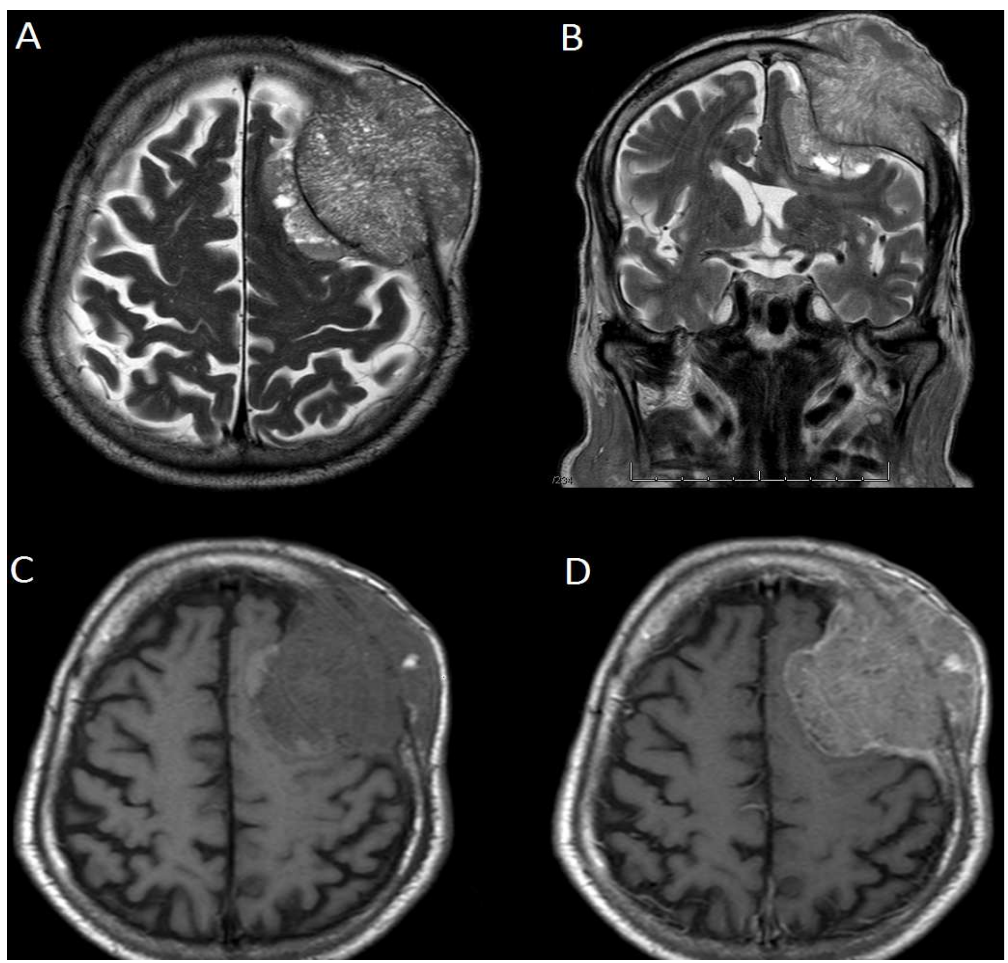

Fig. (2). MRI: T2 Weighted image; axial (a) and Coronal (B) and pre- (C) and post- (D) contrast studies on axial T1 imaging.
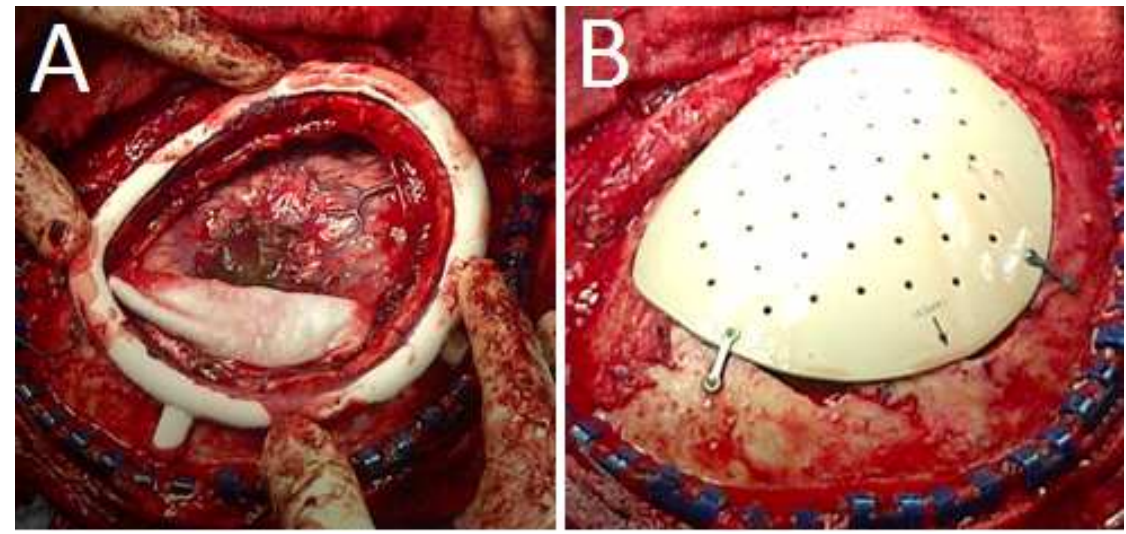

Fig. (3). Intraoperative picture; (A) templates/stencil measuring size of craniectomy. The tumour and dura have been removed and the underlying brain can be seen where the tumor had infiltrated. (B) PEEK implant fitted.

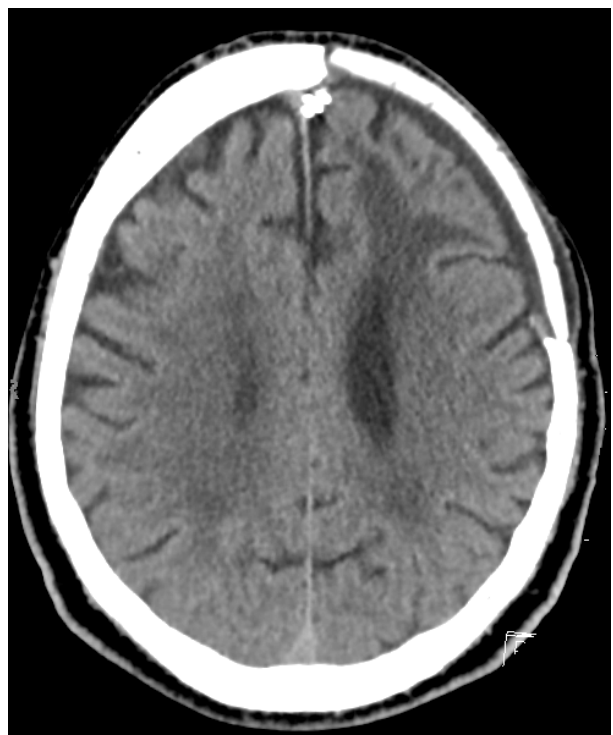

Fig. (4). CT showing expected post surgical changes and the PEEK cranioplasty with no image interferences. 


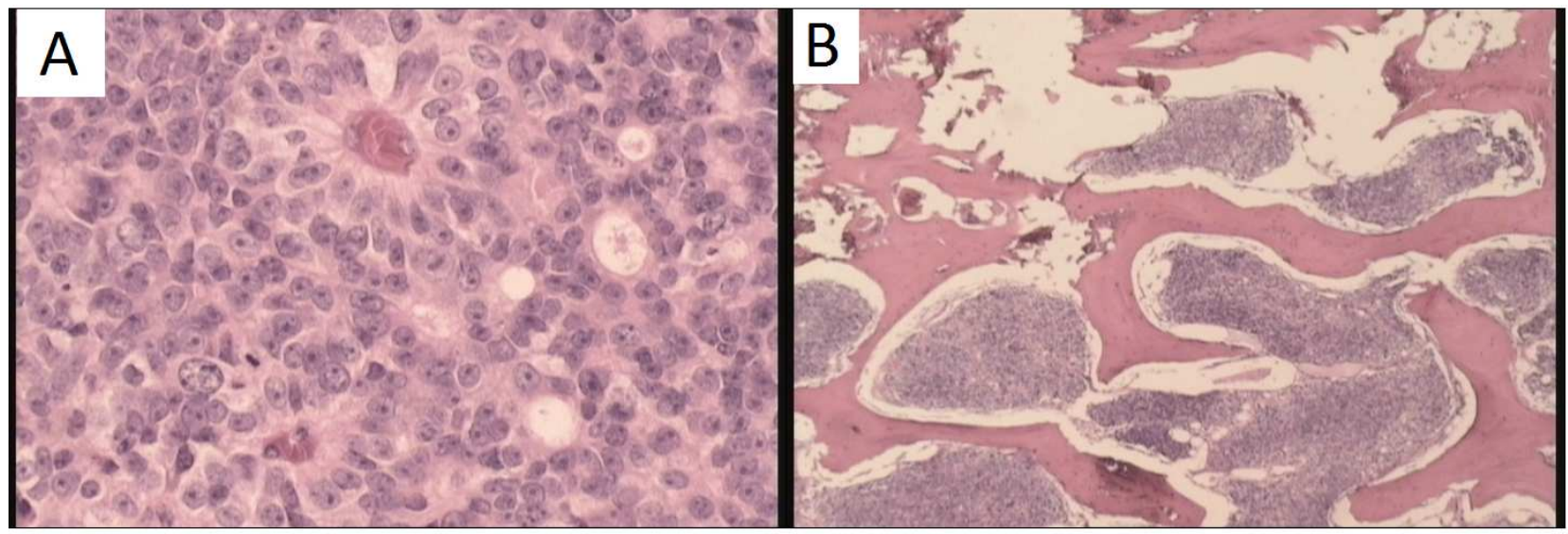

Fig. (5). Histology images showing a haematoxylin and eosin staining revealing the highly cellular nature of the tumor. (A) x 400 magnification. There is a perivascular Rosette in the mid upper field while there are four true rosettes with lumina evident, disposed vertically on the right. (B) x20 magnification. A section of the cancellous bone of the skull showing invasion and replacement of the marrow space by tumor.

author was then able to remotely login into the Swiss engineers workstation using Webex conferencing and was able to plan the PEEK flap in real-time communication with the engineer using advanced computer-aided design software. The precise extent and shape of the implant was planned. At the same time a template was also designed to mark out the extent of the tumour and match the implant to the skull defect. Once the implant was made it was couriered from Switzerland to New Zealand.

During surgery, the scalp and skull flap was marked out based on the template designed. Craniotomy was centred over the tumour and a reasonable margin of bone was removed. This corresponded to the size and conformability of the pre-designed cranioplasty implant Fig. (3). Following tumour excision, the dural defect was reconstructed with artificial dura. A fresh tumour sample was sent to histopathology for immediate macroscopic analysis and appropriate fixation.

Post-operative recovery was uneventful with no new neurological deficit and a CT scan is shown in Fig. (4) Microscopically the sample showed a highly cellular tumour exhibiting abundant rosette formation. Immunostaining showed strong positivity for cytokeratin $8 / 18$ and weak scattered positivity for BerEP4 consistent with carcinoma. Broad spectrum cytokeratin AE1/3 highlighted the luminal surfaces of the true rosettes but with no staining of the tumour cells. A second neuropathologist opinion was obtained from overseas who also agreed that features were typical of neuroendocrine differentiation (Fig. 5).

The patient initially recovered well with mild cognitive impairment. He went through a course of adjuvant radiotherapy and a few months post-operatively, developed generalised tonic-clonic seizures which was treated with anti-epileptics. This was thought to be secondary to surgical scarring or from radiotherapy. Nine months following the surgery he re-presented with new onset weakness, ptosis and diplopia. Full body imaging revealed disseminated malignancy and the patient was treated palliatively.

\section{DISCUSSION}

The common presenting symptom of calvarial metastases is a visible, localized swelling of the skull produced by a growing tumour that erodes the outer table. Tumours can also expand through inner skull tables and apply direct pressure on adjacent brain parenchyma. Rarely, they may be associated with either subdural or epidural hematomas. Focal sensorimotor deficits and seizures occasionally result from progressive inward growth of a calvarial metastatic lesion. Occasionally, a metastatic calvarial mass will compress or occlude a venous sinus. This may lead to elevated intracranial pressure with the clinical symptoms and signs of headache and papilloedema.

Neuroendocrine tumours are a rare group of neoplasms, many of which are benign, that differ in biologic behaviour, histological appearance and response to treatment [1]. They are classified based on tumour grade and differentiation to well and poorly differentiated. Well differentiated tumours arising from the gastrointestinal tract are the most common form but when the primary site is unknown numbers of poorly differentiated tumours with aggressive biology prevail. An unknown primary site of well differentiated neuroendocrine tumours is very rare [1].

Histologically our case was difficult to interpret. The positivity to cytokeratin confirmed that this tumour was a carcinoma but all other lineage specific markers were negative including chromogranins and synaptophysins (the most commonly used neuroendocrine specific markers).

Surgical reconstruction of skull defects is a difficult process that requires multidisciplinary planning and historically involved rejection of implants, poor cosmetic results and re-intervention. In the last 10 years polyetheretherketone (PEEK) has increasingly found a role as a medical implantation device particularly for orthopaedic, maxillofacial and neurosurgical procedures. It is an organic, "shape memory polymer" with highly resistant mechanical and chemical properties. It can withstand the high temperatures used in sterilisation techniques without altering its properties and does not cause artefact to appear on imaging. Computer-designed implants have the advantages of minimizing operative time for preparation, shaping, and insetting. It allows us to prefabricate accurate implants based on 3D images, also known as patient-specific implants (PSI), allowing excellent postoperative cosmetic results [3]. 
Vougioukas et al. in 2004 reported successful use of PEEK implants in fourteen patients who underwent craniofacial reconstruction for congenital deformities, ablative resection of osseous tumors, traumatic injury, and destructive infectious lesions [4]. Our patients' skull defect was as a result of a destructive tumour eroding the left frontal bone through both inner and outer tables. PEEK cranioplasty resulted in good cosmesis with no immediate complication and sufficient preoperative planning allowed tumour removal and alloplastic reconstruction in one procedure, avoiding the undesirable need for temporary skull deformity and re-operation.

Future research may bring about drug impregnated implants to provide local tumour control or bio-degradable implants which may have growth factors impregnated for new bone formation and calvarial reconstruction.

\section{SUMMARY}

Calvarial metastasis is a surgically treatable condition with excellent outcome both cosmetically and clinically for the patient, secondary to the desirable properties of polyetheretherketone. Early Neurosurgical input is recommended for appropriate preoperative planning.

\section{CONFLICT OF INTEREST}

The authors confirm that this article content has no conflicts of interest.

\section{ACKNOWLEDGEMENT}

Declared none.

\section{REFERENCES}

[1] Hainsworth J, Greco F, Strosberg J UptoDate 2011 http://www.uptodate.com/contents/neuroendocrine-cancer-of-unknown-primary-site [last accessed February 2012]

[2] Damianovich D. Scope of the problem in New Zealand. In: Cancer Trials New Zealand. Neuroendocrine tumour workshop 2009. Auditorium, Auckland Hospital, Auckland, New Zealand; p. 4.

[3] Synthes http://www.synthes.com/sites/intl/IntlContent/Files/035.000.376.pdf last accessed February 2012.

[4] Vougioukas VI, Hubbe U, van Velthoven V, Freiman TM, Schramm A, Spetzger U. Neuronavigation-assisted cranial reconstruction. Neurosurgery 2004; 55(1): 162-7.

Received: July 27, 2012

Revised: November 02, 2012

Accepted: November 03, 2012

(C) Merola et al.; Licensee Bentham Open.

This is an open access article licensed under the terms of the Creative Commons Attribution Non-Commercial License (http://creativecommons.org/licenses/by$\mathrm{nc} / 3.0 /$ ), which permits unrestricted, non-commercial use, distribution and reproduction in any medium, provided the work is properly cited. 